\title{
APLICAÇÃO DO TECCOB 2504 NO DISTRIBUIDOR COMO ISOLANTE TÉRMICO E PARA CONTROLE DE INCLUSÕES*
}

Élida Gonçalves das Neves ${ }^{1}$ José Flavio Viana ${ }^{2}$

\section{Resumo}

Os materiais de cobertura de distribuidor são necessários para o isolamento térmico e tem importante interação com o aço líquido durante o lingotamento e logo trazendo uma oportunidade de reduzir o número de inclusões. O objetivo desse trabalho é mostrar os resultados de testes de perda térmica e a capacidade de reter inclusões, através do modelo de inclusões estudado pela Tecnosulfur, do pó de cobertura TECCOB 2504, como uma alternativa para a substituição da palha calcinada.

Palavras-chave: Pó de cobertura; Isolamento térmico; Inclusões não metálicas.

\section{APLICATION OF TECCOB 2504 AS INSULATING POWDER AND INCLUSION ASSIMILATION CAPABILITIES}

\section{Abstract}

The tundish covering materials are required for thermal insulation and has important interaction with the molten steel during the casting and then bringing an opportunity to reduce the number of inclusions. The aim of this work is to show the results of thermal loss testing and the inclusions absorption capability, through the model of inclusions studied by Tecnosulfur of the TECCOB 2504, a tundish cover powder, as an alternative to replace the rice husk ashes.

Keywords: Tundish cover powder; Thermal isolation; Non-metallic inclusion.

1 Engenheira Metalurgista, Bacharel, Engenheira de Processo, Assistência Técnica, Tecnosulfur S/A, Sete Lagoas, Minas Gerais, Brasil.

2 Engenheiro Metalurgista, M.Sc, Gerente Técnico, Gerencia Técnica, Tecnosulfur S/A, Sete Lagoas, Minas Gerais, Brasil. 


\section{INTRODUÇÃO}

Anteriormente, o material mais usado para a cobertura do aço no distribuidor era a palha de arroz devido ao baixo custo. Este material possui boas propriedades de isolamento térmico, permitindo assim uma maior sequência de lingotamento, porém devido ao elevado ponto de fusão, esse material não forma uma camada líquida. Sem a camada liquida, não há absorção de inclusões e nem ocorre a proteção contra a reoxidação, pois é a camada líquida que é impermeável ao ar atmosférico [1].

O alto ponto de fusão também pode possibilitar a formação de crostas sólidas na superfície do distribuidor trazendo dificuldades na medição de temperatura, amostragem de aço e para manuseio de tubo longo nas trocas de panelas.

Outro ponto negativo da palha é que este tipo de material é quimicamente reativo. No caso específico do lingotamento de aços Ultra Baixo Carbono (UBC), uma característica importante no material de cobertura é o teor de carbono, uma vez que esse material não pode contaminar o aço, causando o incremento significativo desse elemento [2].

Devido a estes problemas a palha de arroz foi substituída pela palha calcinada, esta apresentou melhores resultados em relação a variação de carbono, oxigênio, nitrogênio e incorporação de silício pelo banho e a formação de crosta na superfície do banho foi reduzida [2].

Com a maior exigência de aços limpos e necessidade de melhorar o desempenho dos refratários tem-se buscado materiais de cobertura capazes de desempenhar as funções já realizadas pela palha calcinada como o isolamento térmico, e serem melhores quanto a reação com o aço, não alterando a composição química no distribuidor. Além disso espera-se que forme uma camada líquida de escória para absorção de inclusões e proteção contra reoxidação além de não reagir com os refratários para evitar desgaste prematuro nos mesmos.

Os distribuidores desenvolvem hoje um importante papel na limpidez dos aços [3], sendo neles uma das últimas oportunidades de reduzir o número de inclusões. Neste contexto, os pós de cobertura desenvolvem um importante papel atuando na separação de inclusões. Muitos autores consideram o distribuidor como uma importante etapa de controle de limpeza interna do aço. Mesmo os que não consideram o distribuidor como uma etapa em que a limpeza interna pode ser promovida reconhecem a importância deste equipamento para evitar a deterioração desta propriedade [4].

Visando atender a esta demanda, a Tecnosulfur desenvolveu um pó de cobertura capaz de contribuir com a limpidez do metal mantendo a característica de isolamento térmico. O objetivo desse trabalho foi mostrar os resultados de testes de perda térmica e a capacidade de reter inclusões, através do modelo de inclusões estudado pela Tecnosulfur, do pó de cobertura TECCOB 2504.

\section{MATERIAIS E MÉTODOS}

\subsection{Avaliação da Perda Térmica}

Foi realizado teste, em escala piloto no forno de indução com capacidade nominal de $300 \mathrm{~kg}$ de metal liquido para comparar a capacidade de isolamento térmico do produto desenvolvido pela Tecnosulfur, o TECCOB 2504, cuja especificação se 
encontra na tabela 1, e a palha de arroz calcinada. Na tabela 2, segue o descritivo das etapas para realização dos testes.

Tabela 1- Especificação TECCOB 2504

\begin{tabular}{|l|l|}
\hline & $\begin{array}{c}\text { TECCOB 2504 } \\
\text { Composição (\%) }\end{array}$ \\
\hline $\mathrm{CaO}$ & $20 \sim 24$ \\
\hline $\mathrm{CaF}$ & $5 \sim 9$ \\
\hline $\mathrm{MgO}$ & $23 \sim 27$ \\
\hline $\mathrm{SiO}_{2}$ & $13 \sim 23$ \\
\hline $\mathrm{Al}_{2} \mathrm{O}_{3}$ & $11 \sim 23$ \\
\hline
\end{tabular}

Tabela 2- Descritivas das etapas do teste

\begin{tabular}{|l|l|}
\hline Etapa & Descritivo \\
\hline 1 & Fundir o metal. \\
\hline 2 & Elevar a temperatura até uma temperatura próxima de $1450{ }^{\circ} \mathrm{C}$. \\
\hline 3 & $\begin{array}{l}\text { Após atingir a temperatura objetivada o forno de indução era desligado, era feito } \\
\text { a medição de temperatura e o produto (palha ou pó) aplicado e cronometrado } \\
\text { um tempo de } 6,5 \text { minutos para realização de nova medição de temperatura e } \\
\text { quantificado a perda de temperatura neste tempo. }\end{array}$ \\
\hline
\end{tabular}

\subsection{Avaliação da Capacidade da Escória em Remover as Inclusões}

Para a avaliação da capacidade em remover inclusões foi utilizado o modelo apresentado no $45^{\circ}$ Seminário de Aciaria da ABM 2014, que correlaciona a viscosidade com a molhabilidade, conforme figura 1 , sendo a viscosidade (visc) da escória obtida com uso da matemática computacional (FactSage) e a molhabilidade ( $\cos \theta I M S)$ obtida através do cálculo de tensão superficial e interfacial [5]. Neste caso quanto menor a viscosidade e maior a molhabilidade mais a inclusão emerge na escória, evitando assim o seu retorno ao banho.

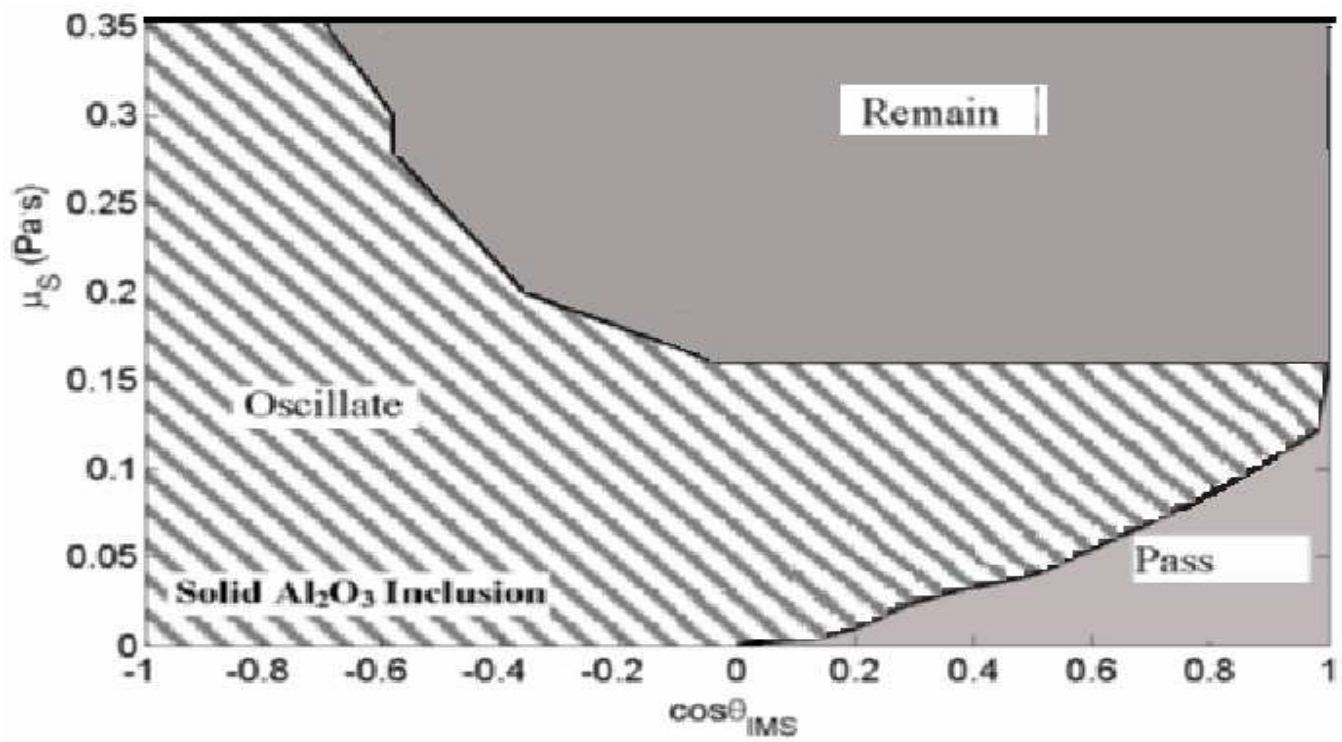

Figura 1 - Comparação do comportamento da inclusão em função da molhabilidade e viscosidade da

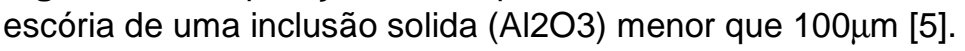




\section{RESULTADOS E DISCUSSÃO}

\subsection{Avaliação da Perda Térmica}

Os resultados dos testes com a palha de arroz calcinada e pó de cobertura estão na tabela 3.

Tabela 3: resultados teste escala piloto

\begin{tabular}{|c|c|c|c|c|c|c|}
\hline Produto & $\begin{array}{c}\text { Quantidade de } \\
\text { produto }(\mathrm{kg})\end{array}$ & $\begin{array}{c}\text { Quantidade } \\
\text { de metal }(\mathrm{kg})\end{array}$ & $\begin{array}{c}\text { Temp. } \\
\text { inicial }\left({ }^{\circ} \mathrm{C}\right)\end{array}$ & $\begin{array}{c}\text { Temp. } \\
\text { final }\left({ }^{\circ} \mathrm{C}\right)\end{array}$ & $\begin{array}{c}\text { Perda Térmica } \\
\left({ }^{\circ} \mathrm{C} / \mathrm{min}\right)\end{array}$ & $\begin{array}{c}\text { Tempo de forno } \\
\text { deligado }(\mathrm{min})\end{array}$ \\
\hline $\begin{array}{c}\text { Palha de } \\
\text { arroz }\end{array}$ & 4,35 & 300 & 1449 & 1322 & 19,54 & 6,5 \\
\hline $\begin{array}{c}\text { TECCOB } \\
2504\end{array}$ & 2,75 & 300 & 1462 & 1427 & 5,38 & 6,5 \\
\hline
\end{tabular}

Após o teste em escala piloto foi calculado a Entalpia de aquecimento para os dois produtos, para avaliar a influência da composição do material no isolamento térmico. Obteve-se que, para a palha de arroz o calor necessário para aquecer este material até $1450{ }^{\circ} \mathrm{C}$ foi de $1.667,58 \mathrm{kcal}$. Enquanto que para aquecer o TECCOB 2504 até a mesma temperatura de $1450{ }^{\circ} \mathrm{C}$ o calor necessário obtido nos cálculos foi de 1.238,82 kcal. Logo temos que, através do cálculo das entalpias de aquecimento, conclui-se que não há diferença significativa entre o calor necessário para aquecer os produtos, portanto a diferença na queda de temperatura está relacionada com o poder de isolamento de cada material.

Foram realizados também, teste industriais com o pó de cobertura para avaliar seu comportamento como isolante no processo industrial, o resultado pode ser visto na tabela 4.

Tabela 4: resultados teste escala industrial

\begin{tabular}{|l|l|l|l|}
\hline Corrida & Temp. inicial $\left({ }^{\circ} \mathrm{C}\right)$ & Temp. final $\left({ }^{\circ} \mathrm{C}\right)$ & Perda Térmica $\left({ }^{\circ} \mathrm{C} / \mathrm{Min}\right)$ \\
\hline 1 & 1498 & 1491 & 0,30 \\
\hline 2 & 1490 & 1480 & 0,67 \\
\hline 3 & 1489 & 1484 & 0,38 \\
\hline 4 & 1482 & 1478 & 0,44 \\
\hline Média & & 0,45 \\
\hline
\end{tabular}

\subsection{Avaliação da Capacidade da escória em Remover as Inclusões}

A avaliação da capacidade de remoção de inclusão foi feita comparando o pó de cobertura TECCOB $2504 \mathrm{com}$ a palha calcinada e um material in natura de referência obtido na literatura, sua composição pode ser obtida na referência 1 . Os dados obtidos com o modelo encontram-se na tabela 5 e foram plotados sobre 0 gráfico do modelo de inclusões, figura 2. 


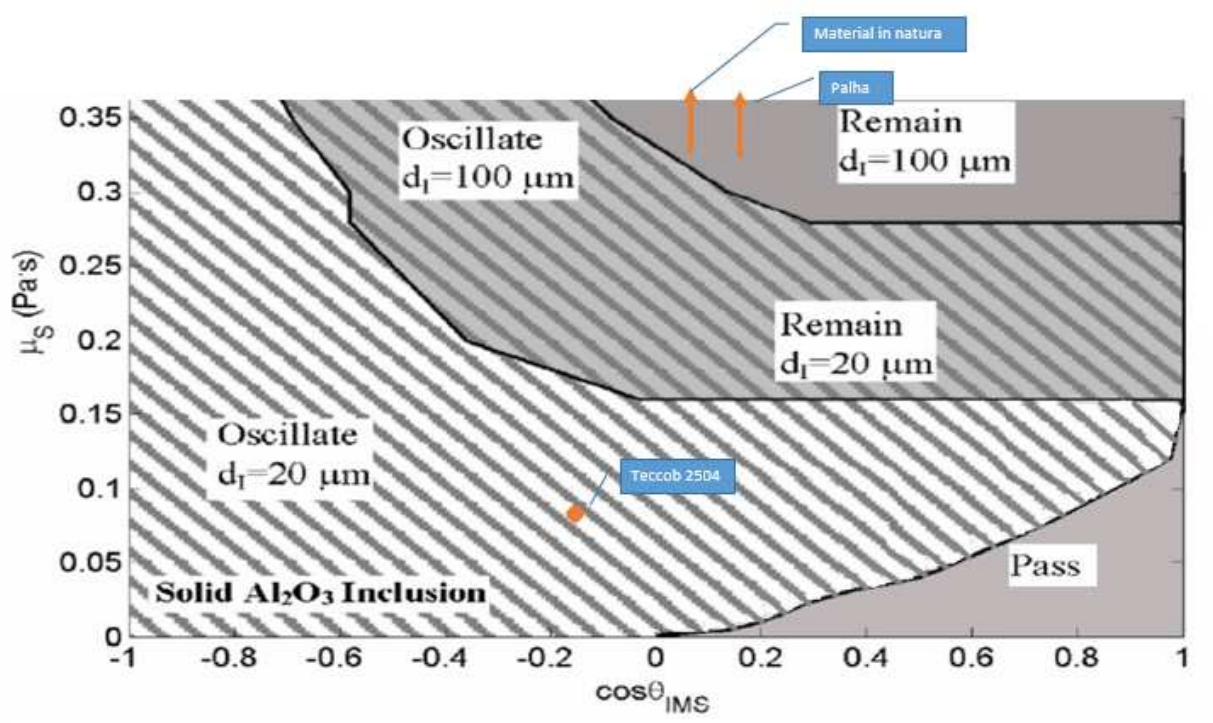

Figura 2 - Comparação do comportamento da inclusão em função da molhabilidade e viscosidade da escória para a palha calcinada, material in natura e TECCOB 2504.

Apesar de possuir molhabilidade maior que a palha e o material in natura, a viscosidade baixa do TECCOB 2504 contribuir para uma melhor capitação de inclusão que os demais produtos. Como o pó de cobertura se mantem fluido durante o lingotamento ele contribui com a absorção de inclusões, com as práticas operacionais padrão de lingotamento e atua na proteção contra a reoxidação, como já mencionado, a camada líquida que é impermeável ao ar atmosférico tornando este um material com melhor performance que os demais analisados.

Tabela 5 - Resultados dos cálculos obtidos com o modelo de inclusão

\begin{tabular}{|c|c|c|c|c|c|c|c|c|c|c|}
\hline Material & visc(Pa.s) & $\sigma \mathrm{m}(\mathrm{N} / \mathrm{m})$ & $\sigma \mathrm{s}(\mathrm{N} / \mathrm{m})$ & $\sigma \mid(\mathrm{N} / \mathrm{m})$ & $\phi M S$ & $\phi S I$ & $\sigma \mathrm{IM}$ & $\sigma \mathrm{MS}$ & $\sigma S I$ & $\cos \theta I M S$ \\
\hline Teccob2504 & 0,085 & 1,50 & 0,383 & 0,609 & $-0,377$ & $-0,660$ & 1,251 & 2,449 & 1,629 & $-0,155$ \\
\hline Palha & $1,95 \mathrm{E}+10$ & 1,50 & 0,262 & 0,609 & $-0,576$ & 0,000 & 1,251 & 2,479 & 0,871 & 0,153 \\
\hline Material in natura & 26,15 & 1,50 & 0,240 & 0,609 & $-0,447$ & $-0,400$ & 1,251 & 2,271 & 1,155 & 0,042 \\
\hline
\end{tabular}

\section{CONCLUSÃO}

O teste em escala piloto com o pó de cobertura mostrou uma redução na perda térmica maior que $70 \%$ quando comparado com a palha calcinada e industrialmente com valores baixos de perda térmica. Devido a boa viscosidade o TECCOB 2504 também se apresenta como melhor captador de inclusões além de fazer uma proteção quanto a reoxidação.

\section{REFERÊNCIAS}

1 Valadares CAG, Bezerra MCC, Sá MLS, Silva ALVC. Uma avaliação dos aspectos termodinâmicos relevantes par o desempenho de um material de cobertura de distribuidor para aços de ultrabaixo carbono. In: Associação Brasileira de Metalurgia, Materiais e Mineração. Anais do $40^{\circ}$ Seminário de Aciaria Internacional; 2009; São Paulo, SP, Brasil: ABM;2009. P.161-172

2 Araujo EPM, Scárdua RN. Utilização de "Palha de arroz calcinada" como material de cobertura de distribuidor para aços UBC na Usiminas. In: Associação Brasileira de 
Metalurgia, Materiais e Mineração. Anais do $31^{\circ}$ Seminário de Fusão, Refino e Solidificação dos Metais; 2000; Vitória, ES, Brasil: ABM;2000. P.581-588

3 Araujo EPM, Scárdua RN. Utilização de "Palha de arroz calcinada" como material de cobertura de distribuidor para aços UBC na Usiminas. In: Associação Brasileira de Metalurgia, Materiais e Mineração. Anais do $31^{\circ}$ Seminário de Fusão, Refino e Solidificação dos Metais; 2000; Vitória, ES, Brasil: ABM;2000. P.581-588

4 Carli R, Del Moro A, Righi C. Tundish covering Materials manufacturing: Real Technology in tundish Metallurgy. Anais do $6^{\circ}$ ECCC; 2008; Riccione, Itália. P. 1-11.

5 Neves EG, Motta MN, Viana JF. Previsão da Limpidez do Aço a partir da Composição Química da Escória. In: Associação Brasileira de Metalurgia, Materiais e Mineração. Anais do $45^{\circ}$ Seminário de Aciaria Internacional; 2014; Porto Alegre, RS, Brasil: ABM;2014. P. 1-6 